\title{
The behavioral investigation of industry 4.0 concept: a research on Twitter
}

\section{Bulent CEKIC}

\section{Haceteppe University, Ankara, Turkey}

\begin{abstract}
:
Aim: This paper is going to investigate how Industry 4.0 concept, behaves in social networks and the context of this innovative approach find a place in time in means of content and sentiment comprising.

Design / Research methods: Expeditious progress in industrialization and information techniques has made great advancement in developing the next span of production technology. Industry 4.0 is an imperative action where the intention is the alteration of modern production through digitalization and profiteering of the capabilities of new advancements. Today, the absence of powerful appliances still feigns a significant impediment for utilizing the ample potential of Industry 4.0. Notably, behavioral approaches are essential for understanding Industry 4.0, which professes novel trials. This paper briefly surveys the area of Industry 4.0 as it relates to behavioral operations by using sentiment analysis and social network analysis methods and tools by describing features of the relationship network either through numerical and visual representation
\end{abstract}

Conclusions/Findings: First of all, it should be presumed that the name Industry 4.0 describes various, fundamentally internet-based developments in manufacturing operations. These advancements do not only have technological but moreover accomplished organizational engagements. Appropriately, a shift from product to service orientation is assumed. Following, the introduction of novel varieties of businesses can be envisioned which embraces new particular functions within the production process sequentially the value-creation networks.

Originality/value of the article: Within the context of the current state of the art in operations management literature, this paper fulfills the gap between behavioral operations and Industry 4.0 context for the researchers both in operations management and behavioral sciences

Keywords: Industry 4.0, Behavioral Operations Management, Social Network Analysis.

JEL: C88, D23, D24, E71, M11, O14, O33

Correspondence address: Bulent CEKIC, Haceteppe University, Ankara, Turkey. E-mail: bulentc@hacettepe.edu.tr.

Received: 11.05.2020, Revised: 07.06.2020, Revised: 11.06.2020, Accepted: 11.06.2020

doi: http://dx.doi.org/10.29015/cerem.878 


\section{Introduction}

The industrial revolution or with a more common name "First Industrial Revolution" is known as a transition period from craft manufacturing to machine production. The wide usage of steam and water power lets people to develop mechanized factory environments. Invention and wide usage of steel and electric power consequently give a lead to mass production transformations in many industries then which this era has been named as "Second Industrial Revolution". This technological innovations also affects the social life in many societies like growing GDPs. It is believed that changing the daily life of people in a good manner, create a positive impression on industry-related thoughts in society. Fostering economic conditions of that era let these adoptions acceptance much easier. Previously invention of the telegraph then Babbage's analytical engine had silently opened the doors of a new communication skill after nearly a century it named as digitalization by Shannon in mid 20th century (see Shannon 1948). The transformation of the analog revolution to a digital one had started and it is called the "Third Industrial Revolution". When society started to meet personal computers and the World Wide Web founded nothing has remained as it is before. Latterly, industrial production systems are being transformed due to a higher level of digitalization, which leads to an intelligent, connected, and decentralized production which points to a novel state, the "Fourth Industrial Revolution". This new level of organization is often called 'Industry 4.0' (Kagermann et al. 2013; Hermann et al. 2016). To sum up and visualize what was told, a detailed timeline drawn by the researchers (Yin et al. 2018) which shows the evolution of production systems among decades should be helpful at Scheme 1. 


\section{Scheme 1. Timeline of industrial revolutions}
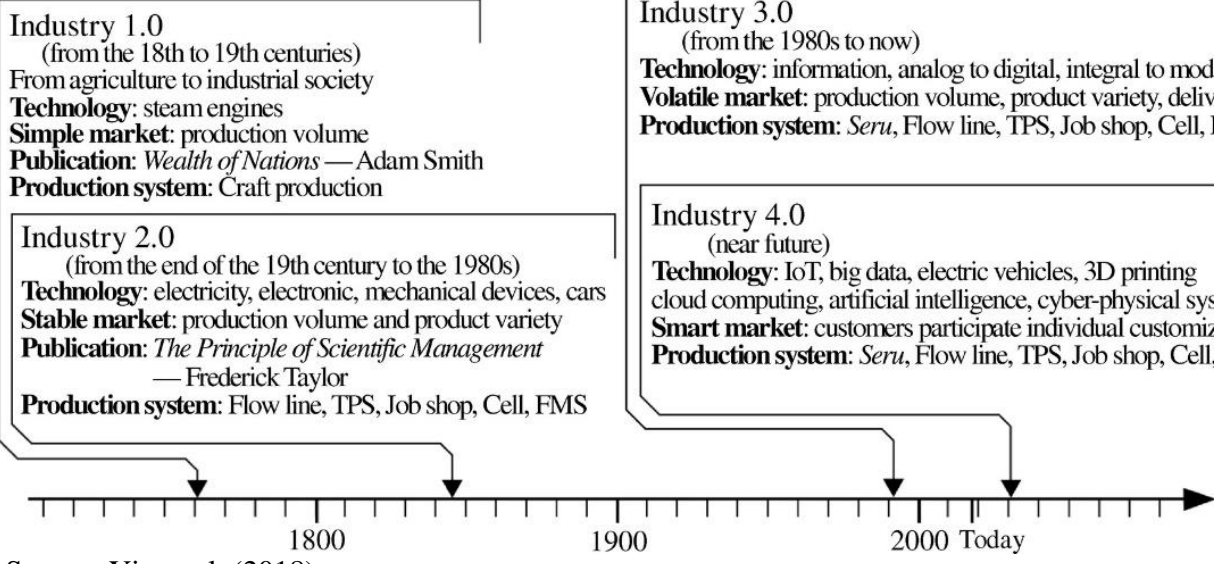

Source: Yin et al. (2018).
Industry 3.0

(from the 1980 s to now)

Technology: information, analog to digital, integral to modular Volatile market: production volume, product variety, delivery time Production system: Seru, Flow line, TPS, Job shop, Cell, FMS

Industry 4.0

(near future)

Technology: IoT, big data, electric vehicles, 3D printing

cloud computing, artificial intelligence, cyber-physical systems

Smart market: customers participate individual customization

Production system: Seru, Flow line, TPS, Job shop, Cell, FMS

Industry 4.0 introduces a pathway to achieve a renewal from mechanical manufacturing to digitized production (Oztemel, Gursev 2020). It is a part of networked and learning artificial organisms, and the Industry 4.0 approach mainly depends on real-time data where innovative processes and novel social architectures are in progress. The researchers' projection about the Industry 4.0 and related components accompanying the journey will have an immense outcome or kind of metamorphosis on social life. The operations community should be provoked spontaneously by this social renewal to advance their process groups to handle the customer requirements and nurture competitive advantage (Oztemel, Gursev 2020). Gathering the data about the behavioral approach of customers or in here the novel technology users is available with social network analysis.

Greater interconnected areas, easier communication of people, and the exposure of lots of information tends society to share their ideas much more courageous ways. Social networks like Facebook, Twitter, Linkedin, etc. has grown up within this motive. Either using WWW or social networking the wider usage of mobile communication, the interactions of individuals, and companies are radically changed. Whether some negative behaviors or effects occurred, the expectations are always positive about these developments. Likewise, a technologically encouraging 
prospect was formed where gadgets will be connected to the internet and make smart cooperations with other objects everywhere at any moment (Lee et al. 2013).

Social Network traffic provokes enormous volumes of data and analysis of this data is important for both organizations and companies to understand the behavior of subscribers. Holding the beat of social network users is important for organizations and individuals to plan their communication, coordinating operations, gathering information, commenting, marketing activities like advertising, and promotion, and organization of social events strategically (Nguyen, Jung 2017; Bello-Orgaz et al. 2016). Piccialli et al. (2018) states that social data can be collected and analyzed for impact, potency, and performance in combination with behaviors. Especially, Twitter gives excellent opportunities for large-scale analytics, which emanate from various aspects: the real-time character of tweets; unpredictable heartfelt reactions; rapid viral spread over the web; etc. Notably, behavioral approaches are essential for understanding Industry 4.0, which professes novel trials (Xu et al. 2018). This paper briefly surveys Industry 4.0 as it relates to behavioral operations by using twitter sentiment analysis and social network analysis tools by describing features of the relationship network either through numerical and visual representation.

\section{Industry 4.0}

The Industry 4.0 idea is depending on the evolution of innovative digital technologies. Respectively the most modern technological advancements, each tool becomes intelligent by the time passes with the internet alliance. The prompt interaction of the internet lets these intelligent devices communicate without human cooperation. Meanwhile, smart agents interact together, activities create a big volume of data, that necessitates to be saved and treated. Industry 4.0 introduces inter-discipliner technologies and offers broad application of AI, CPS, RFID, IoT, additive manufacturing (3D-printing), vertical and horizontal systems integration, smart factories, robotics, simulation, cloud computing, big data and cybersecurity (Xu et al. 2018). While human, machines and sources are vertically connected, the 
businesses are connected horizontally as in a social network created by CyberPhysical Systems (Kagermann et al. 2013; Machado 2020).

As Kagermann et al. (2013) publicly introduced the Industry 4.0 at Hannover Fair in April, 2013 the interest in this topic growth increasingly. The graph seen below proves that enthusiasm as a research interest for the concept itself and its components (Graph 1). This excitement occurred whether the absence of powerful appliances still feigns a significant impediment for utilizing the ample potential of Industry 4.0 that may mean people have a strong positivity about this novel subject.

\section{Graph 1. Google Trend Analysis based on Industry 4.0 and its components (2020, May 1)}

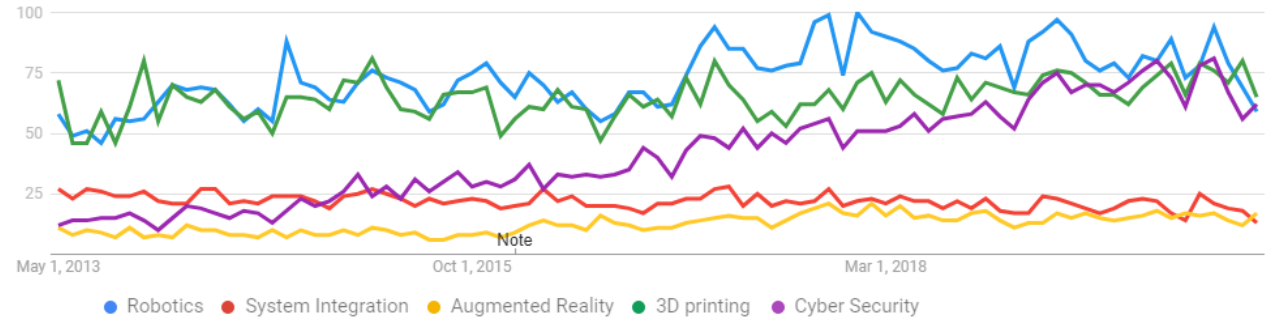

Source: https://trends.google.com/trends/ [01.05.2020]

\section{Social network sentiment analysis}

Presently the active internet user population reaches nearly 4.57 billion people as of May $2020^{1}$. This means nearly 60 percent of the total global population is connecting worldwide as a part of today's information society. Besides 3.81 billion, it means $83 \%$ of active internet users, active social media users connecting and influencing each other. This outrageous growth of internet usage has resulted in users' desire to express their opinions among social media tools like blogging. It is fruitful to extract the mood of people from these opinions (Danescu-Niculescu-Mizil et al. 2009).

\footnotetext{
${ }^{1}$ https://www.statista.com/statistics/617136/digital-population-worldwide/ [02.05.2020].
} 
Microblogging is described as a novel, generally web-based, communication approach where the contributors post their current situation (Java et al. 2007). One of the most popular microblogging services is known as Twitter ${ }^{2}$. This popular microblogging service has a lot of continuous growth in means of users since it launched in October, 2006. Today this social network with 330 million $^{3}$ monthly active users, with more than $40 \%$ of these posting daily, post messages, also called tweets, limited to 280 characters. In Twitter's social network the user intentions help people to connect with similar motives, at the public level, when sharing about their daily activities or opinions about popular subjects (Java et al. 2007). Besides this bursting increase in social media engagement, people and companies start to use the created content in these environments for decision making (Liu 2012). Organizations also started to collect notable feedback about newly released products and services (Go et al. 2009). Certainly, it becomes substantial for companies to hatch their business strategies and plans by considering these opinions, like to understand the feelings about the Industry 4.0 concept in the community. Sentiment analysis would be a great tool for extracting feelings from these unorganized opinions for manipulating many business-intelligence tasks like told (Pang, Lee 2008). The word 'sentiment' 4 is often implied as an emotion inspired by an idea or suggests a settled opinion reflective of one's feelings. The broad discussion about the terminology made at Scherer's study (2005) stated that distinguishing the definitions of opinion, sentiment, feeling, mood and emotion from each other is a challenging point. Sentiment and mood-based empirical analyses on textual data obtained from social media especially Twitter become popular in time (Bollen et al. 2011). In literature, these studies are widely collected under sentiment analysis, and are also called opinion mining (Pang, Lee 2008).

Sentiment analysis is the field of study that analyzes people's behavioral attitudes against entities such as products, services, organizations, individuals, issues, events, topics, and their features (Liu 2012). Sentiment analysis principally concentrates on feelings that prove or mention positive or negative sentiments (Liu

\footnotetext{
${ }^{2}$ https://twitter.com/ [02.05.2020].

${ }^{3}$ https://www.statista.com/statistics/282087/number-of-monthly-active-twitter-users/ [02.05.2020].

${ }^{4}$ https://www.merriam-webster.com/dictionary/sentiment [08.04.2020].
} 
2012). It contributes helpful intuition into online interaction by enabling emotion measurement in those texts. The data gathered from sentiments is used by researchers interested in mining opinions, moods and attitudes, and in-market data, product comparison. Nasukawa and Yi (2003) stated that the machine learning rooted methods are widely used in sentiment analysis, to categorize the feelings, moods, and judgments whether they have positive or negative opinions. Thus, sentiment analysis identifies the affinity of the sentiment phrases with the subject by looking for the polarity and power of the expressions (Nasukawa, Yi 2003).

\section{Methodology}

As it is mentioned one of the key tools for sentiment analysis is machine learning, the techniques used for sentiment categorization are the most commonly naive Bayes, maximum entropy, and support vector machine (Tang et al. 2009). Most sentiment analysis algorithms use simple terms to express the sentiment. However, the cultural factors, linguistic difficulties, and differing contexts prevent researchers from drawing the sentiment accurately. The ANEW is being developed to aid emotion studying researchers to set normative emotional ratings for the English language to figure out a tweet's sentiment (Bradley, Lang 1999). This paper used an adopted dictionary-based approach for determining the sentiment of tweets which is developed by Siddharth and Healey (2011). This ANEW dictionary consists of 1034 rated words along the circumplex model where the arousal and valence are consisting of the dimensions. To plot the map of words matched with the ANEW dictionary using mean valance and arousal, they used the arithmetic mean and normal distribution in the calculation. As the power of the emotion referred by arousal, the degree of negativity and positivity attributed to valence. The circumplex model used at this study which is proposed by Russell (1980) shown in Figure 1, allows us to point out the tweet emotion at any position of valence and arousal. 


\section{Figure 1. Russel's circumplex model}

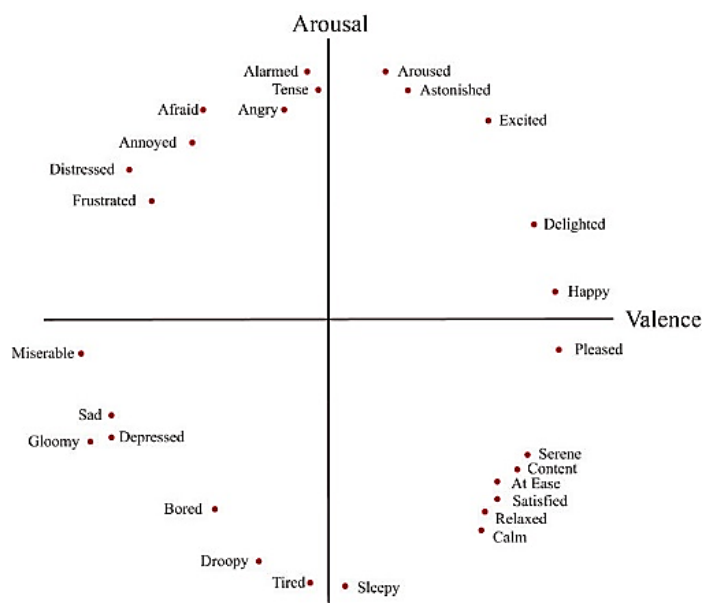

Source: Seo, Huh (2019).

Sentiment analysis provides information about a written material's emotional contextual polarity. ANEW based technique and Deep Learning model are the two methods used in tweet evaluation. Every word in the tweet matched with the ANEW library is mapped in this technique. Then to enhance the mapping this effort enforced to Porter's stemming algorithm (Porter 1980). Further selection decisions were given with matched words by considering the mean valence $\left(\mu_{v}\right)$ and mean arousal $\left(\mu_{a}\right)$ with calculating the average of the valence and arousal for all ANEW library.

Considering this approach, in this paper, the ANEW based methodology developed by Healey and Ramaswamy (2011) is used. Moreover, the data collection, cleaning, parsing, and processing is worked on by the help of a web-based tool ${ }^{5}$ also developed by Healey and Ramaswamy (2011).

${ }^{5}$ https://www.csc2.ncsu.edu/faculty/healey/tweet_viz/tweet_app/ [02.05.2020]. 


\section{Application and findings}

The methodology proposed by Healey and Ramaswamy (2011) converts their open-source code publicly available with a usable web-based tool where you may grab the calculations and visuals easily. The tweet search was done from this site based on Twitter's search API. A virtual database containing hundreds of tweets was processed with the help of this tool. Also, the visuals belong to the Healey and Ramaswamy's (2011) tool. The findings of the Industry 4.0 based search and the terms affection on Twitter users are shown and discussed below.

Twitter keyword search is done with the term "Industry 4.0" and as a mention "\#industry40" results with hundreds of findings limited with the tools search time interval. The first figure (Figure 2) below is a sample ANEW dispatch for a just one tweet in part. As was told in the previous methodology section, the valence and arousal scores, for the terms 'industry', 'emerges' and 'internet" are [5.30, 4.47], $[2.72,6.43]$ and $[6.68,5.44]$ calculated respectively. The overall valence score $\mu_{\vartheta}=5.07$ and the overall arousal $\mu_{\propto}=5.45$ has found for this particular sample tweet. For ANEW it is calculated a standard deviation, shown in the figure also, for every term's valence and arousal, which explains the expansion of potential valences and arousals for the terms used in divergent contexts.

\section{Figure 2. Tweet details}

May $6,2: 32 p m$ The fourth Industrial revolution emerges from Al and
the Internet of Things https://t.co/qSAavfZW
$v=5.07, a=5.45$
$\begin{aligned} & \text { industri, } v=[\mu: 5.3, \sigma: 1.61], a=[\mu: 4.47, \sigma: 2.43], f a=171 \\ & \text { emerg, } v=[\mu: 2.72, \sigma: 1.23], a=[\mu: 6.43, \sigma: 2.73], f a=19 \\ & \text { internet, } v=[\mu: 6.68, \sigma: 1.53], a=[\mu: 5.44, \sigma: 2.73], f a=18\end{aligned}$

Source: https://www.csc2.ncsu.edu/faculty/healey/tweet_viz/tweet_app/ [06.05.2020].

Next, following the method of the normal distributions by using the means of valence and arousal and for the words listed in ANEW, the standard deviations were calculated. Following, these listed words' computed normal distributions added into a cumulative distribution by using the cumulative distribution functions. This helps us in finding the coordinates, which are the maximum of the results that come out 


\section{Bulent CEKIC}

from the cumulative distribution functions. The x-coordinate results with $\mu_{\vartheta}$ and $\mu_{\alpha}$ where the mean score is maximum representing the y-coordinate. After that, to map them on the scale, like seen below (Figure 3) we need to normalize $\mu_{\vartheta}$ and $\mu_{\propto}$.

\section{Figure 3. Sentiment map visual}

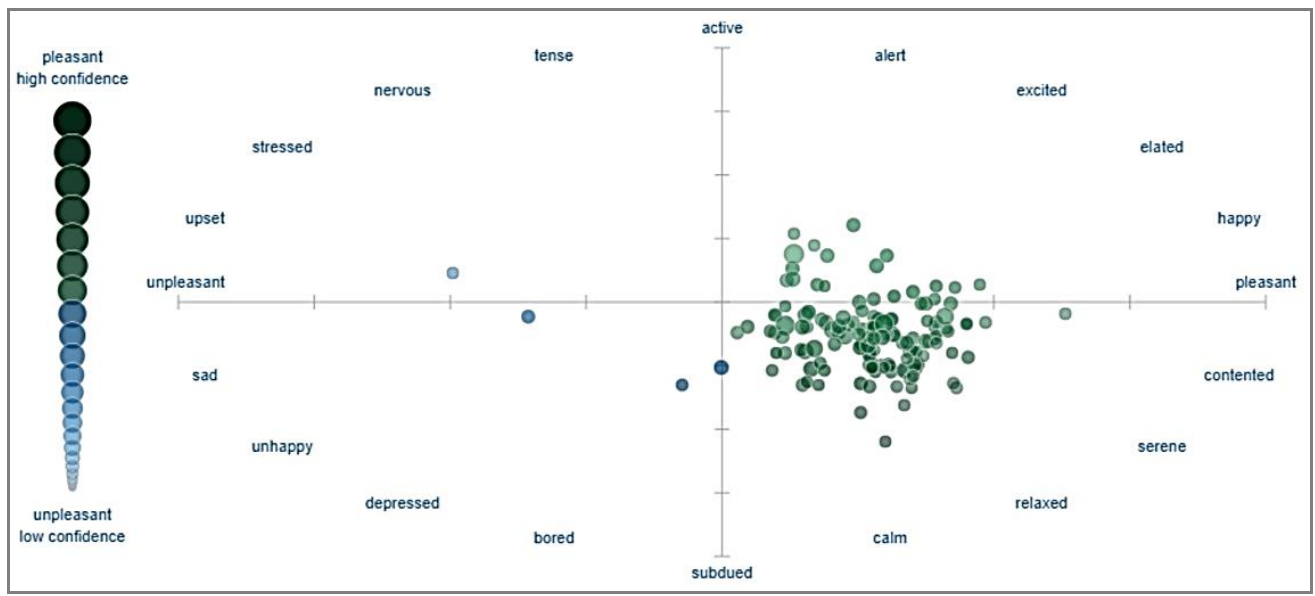

Source: https://www.csc2.ncsu.edu/faculty/healey/tweet_viz/tweet_app/ [06.05.2020].

The above graph (Figure 3) is the visualized form of how "Industry 4.0" related tweets distributed among the scale's axis. This geographical location expresses the overall sentiment of the tweets. In this particular search, we can figure out that the attitude towards this new development is pleasant but subdued. Besides, the transparency of the visuals, which represents the power of confidence, are tending as opaque that tells us the estimates are assured. 
Figure 4. Tweets affinity graph

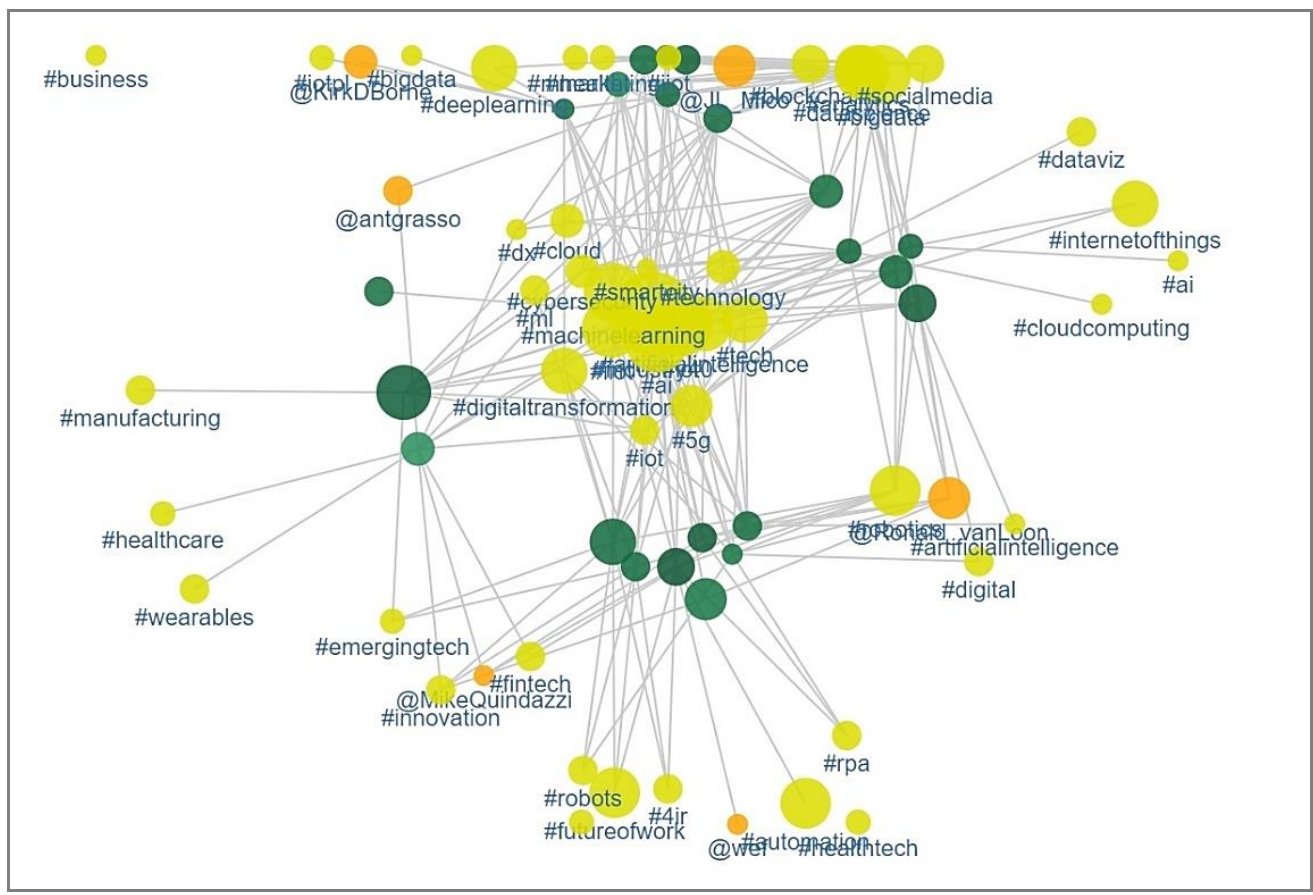

Source: https://www.csc2.ncsu.edu/faculty/healey/tweet_viz/tweet_app/ [06.05.2020].

The affinity graph visualizes the frequency of tweets together with relationships or closeness between each other. As indicated in related literature (Liao et al. 2017; Xu et al. 2018; Oztemel, Gursev 2020), the relationship between Industry 4.0 and its components is obvious and strong. The strongly linked subjects should easily be observed from the graph like digital transformation, cloud computing, innovation, automation, robots, IoT, AI, cybersecurity, machine learning, healthcare, etc. Furthermore, the following word cloud distributed among the emotional circumplex model both indicates the tweet owners feelings and the sentimental status of the hashtags they used when writing about subject Industry 4.0. 
Figure 5. Tweets tag cloud

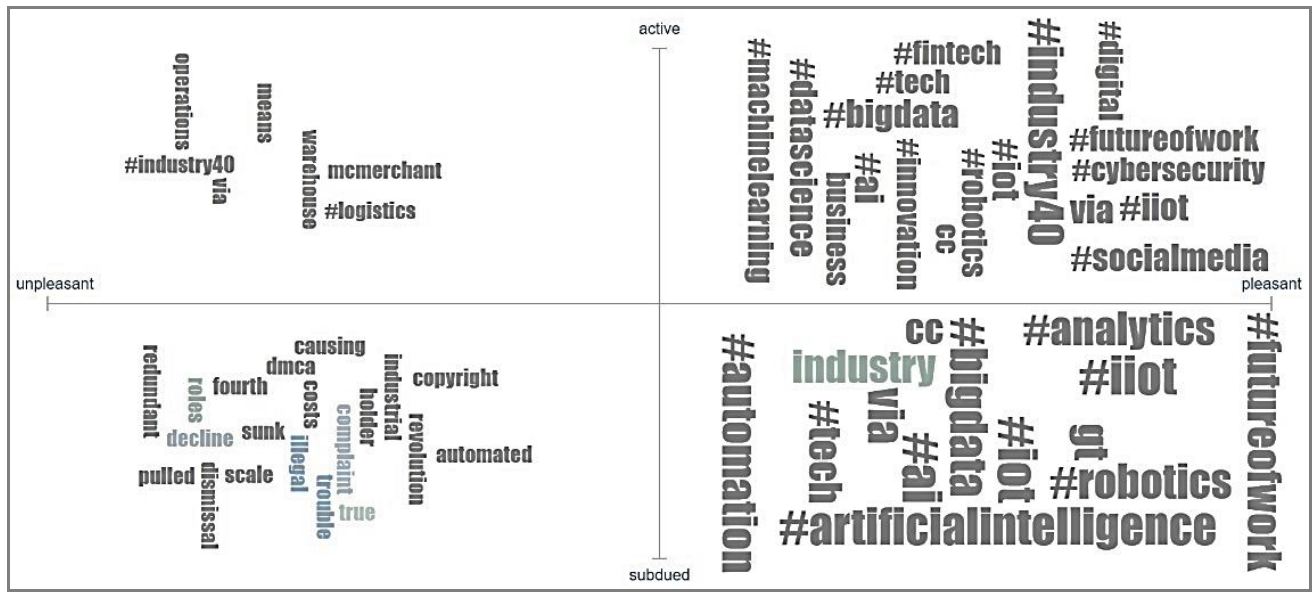

Source: https://www.csc2.ncsu.edu/faculty/healey/tweet_viz/tweet_app/ [06.05.2020].

\section{Concluding remarks}

Human being tends such innovative approaches in all ages and eras. The findings show no hesitation that the interest of operations decision-makers about digital transformation in general but Industry 4.0 in specific, is high. The sentiment analysis, performed in this paper, approves this positivity within the Twitter data. The calmness about the 'Industry 4.0' resulted from the sentiment map (Figure 3) should be explained with the absence of powerful appliances still feigns a significant impediment for utilizing the ample potential of Industry 4.0. Also, the resistance among the employee side still not explained in detail. The other approaches associated with Industry 4.0 like Work 4.0 and Society 5.0 creates pressure on both working and social life. Hence, rather the dullness or sometimes negativity pops up in the social media texting.

This social network investigation is important because of the decision-making algorithm's dependency on the surrounding environment. We couldn't take apart the managers decisions free of their imposed and effected by their network. Especially the complaints about the company's products and/or services that are fashionably 


\section{THE BEHAVIORAL INVESTIGATION OF INDUSTRY 4.0 CONCEPT}

favorable on the customer side become extremely important. Hence, the negative opinions weigh more than positive ones (Dave et al. 2003).

This study should be performed with a larger database to be more precise. Pajek, UciNet, NodeXL, and many more social network analysis tools will be helpful in this manner. Within this expansion, not only the Industry 4.0 attachment but also its environment with the core components will become understandable sentimentally.

\section{Acknowledgments}

The author would like to thank the anonymous reviewers for their careful reading and many insightful contributions.

\section{References}

Bello-Orgaz G., Jung J.J., Camacho D. (2016), Social big data. Recent achievements and new challenges, "Information Fusion", vol. 28, pp. 45-59.

Bollen J., Mao H., Pepe A. (2011), Modeling public mood and emotion. Twitter sentiment and socioeconomic phenomena, in: Fifth International AAAI Conference on Weblogs and Social Media, July.

Bradley M.M., Lang P.J. (1999), Affective norms for English words (ANEW). Instruction manual and affective ratings, Technical report C-1, The Center for Research in Psychophysiology, University of Florida, https://www.uvm.edu/pdodds/teaching/courses/2009-08UVM300/docs/others/everything/bradley1999a.pdf [13.06.2020].

Danescu-Niculescu-Mizil C., Kossinets G., Kleinberg J., Lee L. (2009), How opinions are received by online communities. A case study on amazon.com helpfulness votes, in: Proceedings of the 18th International Conference on World Wide Web, April, pp. 141-150.

Dave K., Lawrence S., Pennock D.M. (2003), Mining the peanut gallery. Opinion extraction and semantic classification of product reviews, in: Proceedings of the 12th International Conference on World Wide Web, May, pp. 519-528.

Go A., Bhayani R., Huang L. (2009), Twitter sentiment classification using distant supervision, CS224N Project Report, Stanford, https://cs.stanford.edu/people/alecmgo/papers/TwitterDistantSupervision09.pdf [13.06.2020].

Healey C.G., Ramaswamy S.S. (2011), Visualizing Twitter sentiment, Sentiment viz, https://www. csc. ncsu. edu/faculty/healey/tweet_viz/tweet_app/ [06.05.2020]. 
Hermann M., Pentek T., Otto B. (2016), Design principles for industrie 4.0 scenarios, in: 2016 49th Hawaii International Conference on System Sciences (HICSS). Conference Proceedings, Koloa (HI), pp. 3928-3937.

Java A., Song X., Finin T., Tseng B. (2007), Why we twitter. An analysis of a microblogging community, in: International Workshop on Social Network Mining and Analysis, August, Springer, Berlin - Heidelberg, pp. 118-138.

Kagermann H., Helbig J., Hellinger A., Wahlster W. (2013), Recommendations for implementing the strategic initiative Industrie 4.0. Securing the future of German manufacturing industry; final report of the Industrie 4.0 Working Group, Forschungsunion.

Lee J., Lapira E., Bagheri B., Kao H.A. (2013), Recent advances and trends in predictive manufacturing systems in big data environment, "Manufacturing Letters", vol. 1 no. 1, pp. 38-41.

Liao Y., Deschamps F., Loures E.D.F.R., Ramos L.F.P. (2017), Past, present and future of Industry 4.0. A systematic literature review and research agenda proposal, "International Journal of Production Research”, vol. 55 no. 12, pp. 3609-3629.

Liu B. (2012), Sentiment analysis and opinion mining, "Synthesis Lectures on Human Language Technologies", vol. 5 no. 1, pp. 1-167.

Machado C.G., Winroth M.P., Ribeiro da Silva E.H.D. (2020), Sustainable manufacturing in Industry 4.0. An emerging research agenda, "International Journal of Production Research", vol. 58 no. 5, pp. $1462-1484$.

Nasukawa T., Yi J. (2003), Sentiment analysis. Capturing favorability using natural language processing, in: Proceedings of the 2nd International Conference on Knowledge Capture, October 2325, Sanibel Island, pp. 70-77.

Nguyen D.T., Jung J.E. (2017), Real-time event detection for online behavioral analysis of big social data, "Future Generation Computer Systems", no. 66, pp. 137-145.

Oztemel E., Gursev S. (2020), A taxonomy of Industry 4.0 and related technologies, in: Industry 4.0. Current status and future trends, Hamilton Ortiz J. (ed.), IntechOpen, https://www.intechopen.com/books/industry-4-0-current-status-and-future-trends [13.06.2020].

Oztemel E., Gursev S. (2020), Literature review of Industry 4.0 and related technologies, "Journal of Intelligent Manufacturing", vol. 31 no. 1, pp. 127-182.

Pang B., Lee L. (2008), Opinion mining and sentiment analysis, "Foundations and Trends in Information Retrieval", vol. 2 no. 1-2, pp. 1-135.

Piccialli F., Benedusi P., Amato F. (2018), S-InTime. A social cloud analytical service oriented system, "Future Generation Computer Systems", vol. 80, pp. 229-241.

Porter M.F. (1980), An algorithm for suffix stripping, "Program”, vol. 14 no. 3, pp. 130-137.

Ramaswamy S.S. (2011), Visualization of the sentiment of the tweets, Master's Thesis, North Carolina State University, Raleigh, NC. 


\section{THE BEHAVIORAL INVESTIGATION OF INDUSTRY 4.0 CONCEPT}

Russell J.A. (1980), A circumplex model of affect, "Journal of Personality and Social Psychology", vol. 39 no. 6 , pp. 1161-1178.

Scherer K.R. (2005), What are emotions? And how can they be measured?, "Social Science Information", vol. 44 no. 4, pp. 695-729.

Seo Y.S., Huh J.H. (2019), Automatic emotion-based music classification for supporting intelligent IoT applications, "Electronics", vol. 8 no. 2, p. 164.

Shannon C.E. (1948), A mathematical theory of communication, "Bell System Technical Journal", vol. 27 no. 3, pp. 379-423.

Tang H., Tan S., Cheng X. (2009), A survey on sentiment detection of reviews, "Expert Systems with Applications", vol. 36 no. 7, pp. 10760-10773.

Xu L.D., Xu E.L., Li L. (2018), Industry 4.0. State of the art and future trends, "International Journal of Production Research”, vol. 56 no. 8, pp. 2941-2962.

Yin Y., Stecke K.E., Li D. (2018), The evolution of production systems from Industry 2.0 through Industry 4.0, "International Journal of Production Research", vol. 56 no. 1-2, pp. 848-861. 